\title{
Development of Mode-matching Techniques to Efficiently Model Multi-mode Horns with Non-PEC Walls
}

\author{
Joseph Brennan, Marcin Gradziel, and Neil Trappe \\ Department of Experimental Physics, Maynooth University, Collegeland, Maynooth Co. Kildare, Ireland
}

\begin{abstract}
In the design and analysis of millimetre wave components imperfections of the guide wall material are frequently overlooked and PEC (Perfect Electric Conductor) behaviour is assumed to simplify the problem. These imperfections could be due to the large yet finite wall conductivity or the surface roughness associated with manufacturing processes. In reality, when non-PEC walls are considered, they have a non-negligible effect on mode attenuation and mode mixing, particularly at guide discontinuities An extension of the well-established Mode-Matching method to include non-PEC materials is investigated in this paper. Simply put, Mode-Matching is a method used to determine the scattering coefficients at guide discontinuities. Here the transverse fields on both sides of the step are "matched" such that we have conservation of complex power for incident modes. The additional boundary conditions imposed by the nonPEC walls are considered as perturbations to the PEC solutions. In uniform guides this opens further channels of mode mixing, as opposed to modes bijectively matching; and introduces mechanisms for attenuation which exist due to the necessary surface impedance on the guide walls. While at junctions, further mechanisms for attenuation exist due to the surface impedance on the overlap region of the guides. A volumetric finite element solver is used as a benchmark for the verification of the method. However, for larger components the simulation time required for the finite element solver becomes impractical. Hence, the Mode-Matching description provides a near perfect description of the effect of the non-PEC walls at a fraction of the computational cost when compared to the finite element solver.

We model two types of manufactured horn antennae; a conical cylindrical and corrugated cylindrical horn each with $100+$ segments. A PEC solution is used as a benchmark for simulations where the physical surface parameters are included. This highlights the effect of including loss in the guide on the losses in individual horn modes. With the increasing detail of horn geometries, the computational effort and simulation time required to effectively model them also increases. We also discuss methods of parallelisation and hardware acceleration of the Mode-Matching code to deal with the increasing computation demand on electromagnetic simulations. These methods are developed in OpenCL to ensure portability of the software across different hardware architectures.
\end{abstract}

\section{INTRODUCTION}

The modal method or mode-matching techniques are employed to accurately model, characterise, and aid in the optimisation of single and multimode waveguide structures at millimetre and submillimetre wavelengths. Mode-Matching is a method used to describe the transmission and reflection at waveguide discontinuities or junctions. By considering the modal fields on both sides of the discontinuity, the power coupling integral can be obtained by performing a surface integral over the surface element at the junction. These techniques are well established and have contributed greatly to the development of multiple millimetre wave instruments' detector array systems [1]. The results from the modal analysis of waveguide systems is well-known to be in excellent agreement with alternative methods including finite element solvers, which solve Maxwell's equations for the entire structure. Here, the mode-matching methods have a significant advantage in terms of computational effort, especially for electrically large systems where finite element techniques struggle.

In those implementations of the mode-matching method, the treatment of the bounding conducting walls of the waveguide structures have been purely PEC (Perfect Electric Conductor). It is clear that the accuracy of the model is more than adequate for modelling these structures without losses on the bounding walls. However, there remains a question mark over the description of multimoded or highly over-moded waveguides. By including large yet finite conductivity in our description of the mode-matching method, we aim to more accurately describe the effect of these losses, which are expected to have a more significant effect for the over-moded guides [2]. The effect of including physical material parameters gives non-negligible effects when considering either the interface between guide components or in cavity sections. If these can be modelled effectively then 
the manufactured components can be well characterised before any experimental measurements are performed with the device.

For the full description of a lossy waveguide structure to be obtained, the complete description of the constituent elements must be found. As for PEC structures, cascading methods are employed to construct the more elaborate geometries which are of interest. Therefore, the methods to describe how the modal fields exist in uniform lossy elements and the effect the lossy walls will have on the boundary conditions at discontinuities between uniform elements must be obtained. In over-moded uniform lossy sections, the effect of interfering currents at the bounding wall due to multiple modes present must be considered. The relative phases of these guide modes can have a strong effect on the power dissipated in waveguide. In general it is not always correct to consider the individual losses [2]. Thus, it can be taken that the power dissipated by modes at the guide walls must be taken together. As a consequence, the correct prediction involves a coupling of the modes within the uniform lossy section. To consider the effect of the lossy walls at discontinuities, the familiar conservation of complex power techniques used in the general approach to mode-matching between PEC guide sections are employed. Due to the finite wall conductivity on the overlap region in the larger guide section, we have a modification to the boundary conditions. In general, this has minimal impact on the scattering parameters, where quite high conductivity is applied [3]. However, this effect becomes more apparent with resonant type structures. In the following section we aim to describe the implementation of these methods and provide verification of their correctness.

\section{LOSSES IN UNIFORM AND DISCONTINUOUS ELEMENTS}

\subsection{Uniform Lossy Guide}

The inclusion of finite wall conductivity on the boundary walls requires the surface impedance $\left(Z_{s}\right)$ to be included in our description of the uniform lossy guide section. An additional boundary condition on the tangential component of the electric field exists along an interface between two media, one of which has a large yet finite conductivity and the other a lossless isotropic medium [4]. This is illustrated in Fig. 1, where medium 2 is a good conductor whose surface exhibits a surface impedance given in (1).

$$
Z_{s}(\omega)=R_{s}+j X_{s}=(1+j) \sqrt{\frac{\omega \mu}{2 \sigma_{1}}}
$$

At the surface there exists a linear current density $\bar{J}_{s}$ related to the tangential magnetic field in medium 1 by $\bar{J}_{s} \cong \hat{n} \times \bar{H}_{2}$. As the conductivity is finite, the most intense current density resides at the surface and diminishes exponentially as observations are made deeper into the conductor. Additionally, the electric field intensity along the boundary of the two media cannot be zero [4]. Thus, we can write that the tangential component of the electric field in medium 1, along the boundary, is linked to the current density $J_{s}$ and the tangential component of the magnetic field by $(2)$.

$$
\bar{E}_{t, 1}=Z_{s}(\omega) \bar{J}_{s}=Z_{s}(\omega) \hat{n} \times \bar{H}_{1}=\hat{n} \times \bar{H}_{1}(1+j) \sqrt{\frac{\omega \mu}{2 \sigma_{2}}}
$$

Where $\hat{n}$ is the vector normal to the contour of the interior of the waveguide, and there exists a vector $\hat{t}$ which is tangential to the contour such that:

$$
\hat{n} \times \hat{t}=\hat{z}, \quad \hat{t} \times \hat{z}=\hat{n}, \quad \hat{n} \times \hat{z}=\hat{t} .
$$

In a uniform guide the normal to the surface is opposite to the contour normal. Hence, the $z$ component of the magnetic field is always tangential to the surface of the guide. As such, it is

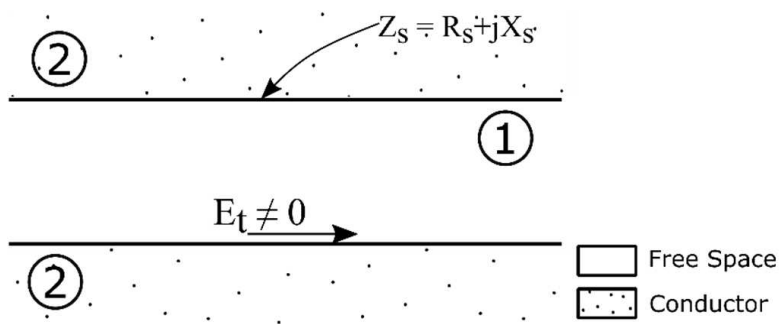

Figure 1. Shows the existence of a non-zero tangential E-field at the boundary due finite wall conductivity. 
linked to the component of the electric field parallel to $\hat{t}$. Since $\hat{t}$ is orthogonal to $\hat{z}$ we need only consider $E_{t}$. In a similar manner, the component of the magnetic field parallel to $\hat{t}$ is linked to the $z$ component of the electric field. It can be said that the lossy waveguide solution is a perturbation of the PEC solution where the transverse electric field component at the boundary is produced by the magnetic interaction with the non-PEC walls. These additions must be taken together as when taken individually the net result of these electric field additions appears to be zero [2].
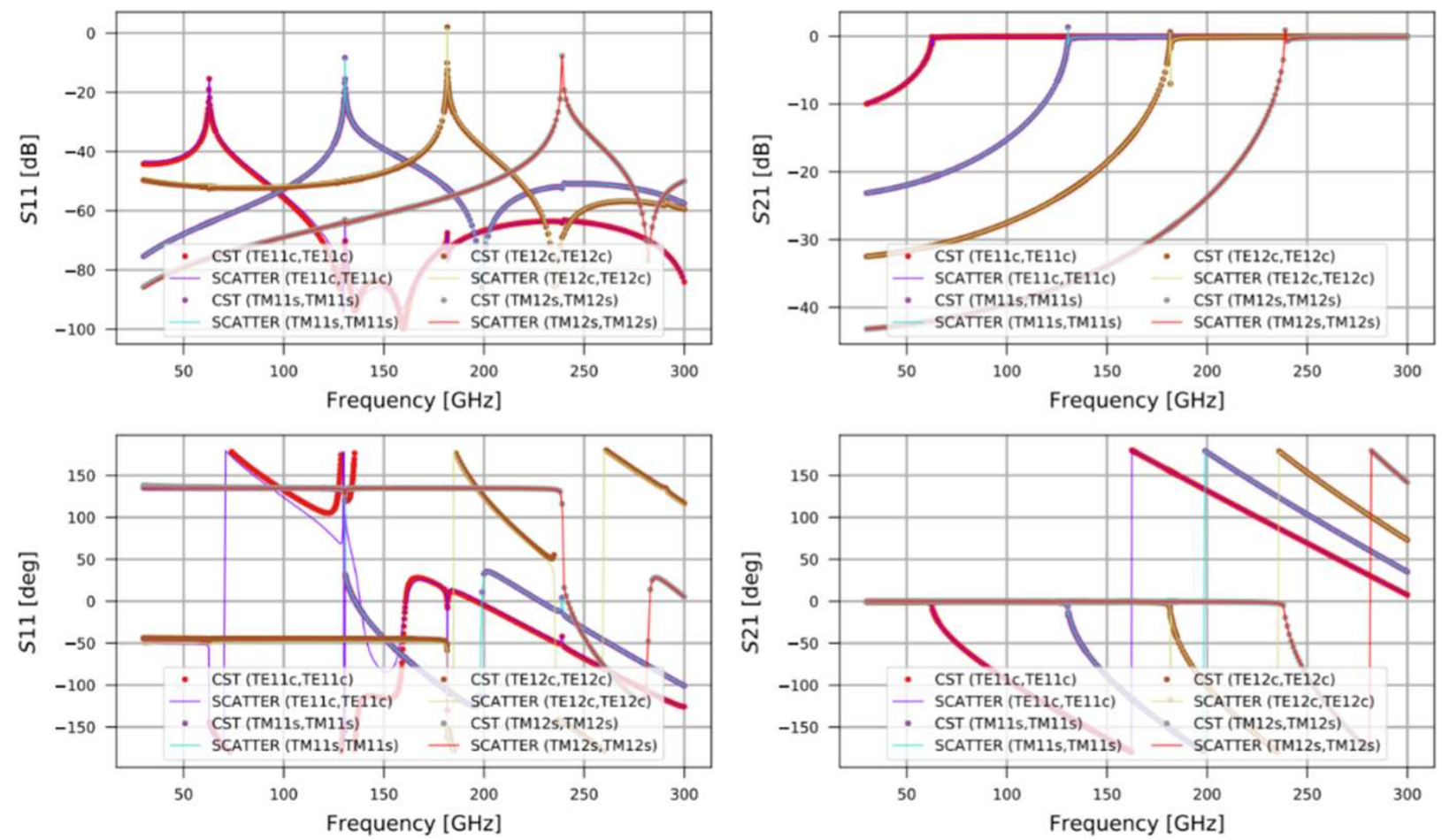

Figure 2. The scattering parameters both reflection and transmission values are shown for various combinations of modes. The results are in perfect agreement with the same simulation results from CST.

Consider a uniform cylindrical guide section of radius $1.4 \mathrm{~mm}$, length $1.0 \mathrm{~mm}$ and wall conductivity of $\sigma=5.4 \times 10^{4} \mathrm{~S} / \mathrm{m}$. The low value of conductivity is used to exaggerate the losses due to the finite conductivity in our uniform guide section. An identical system is modelled in CST and the results for both simulations are shown in Fig. 2, over a frequency range of 30-300 GHz. Excellent agreement is observed between the results from the two simulations. Additional observation can be made with regard to the cross-coupling of orthogonal modes in the guide. In contrast to a PEC guide where modes strictly couple to themselves only. Observed in Fig. 2, we also see that there are non-zero components of reflection in our lossy guide and that there is a small reduction to the overall transmission in each mode. Again, in contrast to the PEC guide no reflection would be observed and full transmission would be expected.

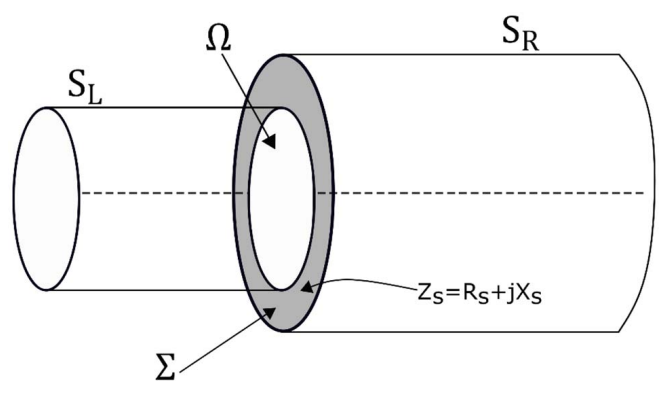

Figure 3. Junction between two guides, with the regions of interest for the boundary conditions highlighted. 


\subsection{Lossy Junction}

Assuming a junction between two guides Fig. 3, which we will commonly refer to as 'Left' and 'Right' or $L$ and $R$ at $z=0$. We say that these sections have a cross section defined as $S_{L} \& S_{R}$ respectively such that $S_{L} \subset S_{R}$, the common cross section of the two guides in $\Omega=S_{L} \cap S_{R}$ and the overlap region is referred to as $\Sigma=\left(S_{L} \cup S_{R}\right)-\Omega$. In this situation, $\Sigma$ defines the wall of the waveguide, which has good yet finite conductivity. The general boundary conditions for transverse fields for the lossy junction require:

$$
\bar{E}_{L}=\bar{E}_{R} \text {, on } \Omega \quad \bar{H}_{L}=\bar{H}_{R}, \text { on } \Omega \quad \bar{E}_{R} \cong Z_{w} \hat{z} \times \bar{H}_{R} \text {, on } \Sigma
$$

The same definition of the surface impedance from the previous section used. The interface between two guide components with PEC walls would require that fields remain continuous across the interface, while in the overlap region the electric field must be zero away from the common interface [3]. However, since there exists a finite wall conductivity at the overlap region this is no longer the case and a new boundary condition for the overlap region must be considered. This results in the necessary existence of an additional electric field on the right hand side of the junction which must be considered. The additional electric field is a result of the interaction of the magnetic field with the lossy overlap region resulting in a similar perturbation to the electric field as observed in the lossy uniform guide case.

Consider the junction between two cylindrical waveguides with radii $1.4 \mathrm{~mm}$ and $1.7 \mathrm{~mm}$ both with equal length of $1 \mathrm{~mm}$ and wall conductivity of $\sigma=5.4 \times 10^{4} \mathrm{~S} / \mathrm{m}$. Again, low conductivity is used to exaggerate the effects of the lossy walls on the transmission and reflection. As conductivity tends toward high conductivity material, i.e., copper or brass, there is negligible difference to the results from a similar PEC walled structure. Again by contrasting the results against an identical simulation performed in CST, we see in Fig. 4 that there is excellent agreement between the two simulations.
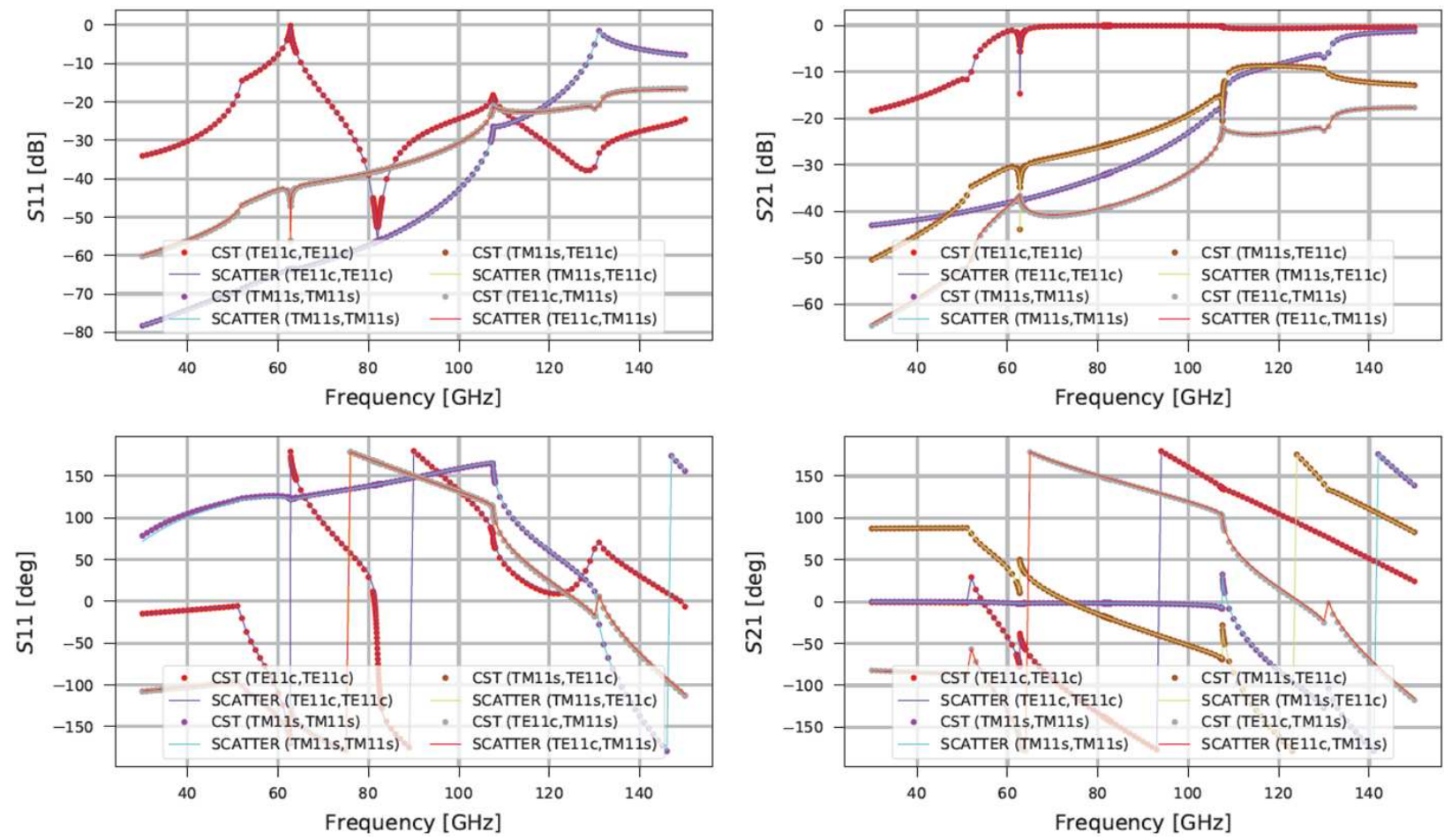

Figure 4. Results for selected modes in $1.4 \mathrm{~mm}$ circular to $1.7 \mathrm{~mm}$ circular guide junction, with no offset. Conductivity was $\sigma=54000 \mathrm{~S} / \mathrm{m}$ radial mode orders were included in the Scatter TNG model.

\subsubsection{Rough Surfaces}

The lossy model considered for the uniform guide section and junction requires only knowledge of the surface impedance of the bounding wall. A physical surface roughness model, detailed in [5], is adapted to allow for the effect of roughness to be modelled in our mode-matching implementation of lossy guides and junctions. For this model to work some conditions must be imposed; 
- Wavelength $\lambda \gg$ conductor dimensions $w, t$

- conductor dimensions $w, t \gg \operatorname{skin} \operatorname{depth} \delta$

- skin depth $\delta \leq$ surface roughness $R q$

Roughness results in an increase to the overall impedance of the guide walls which can be easily included in the simulations. The conductivity of the material is assumed to be location dependent $\sigma(x)$, thus requiring that there exists a gradient before the bulk conductivity of the material is achieved. Solving the Helmholtz equation with this assumption gives the rough surface impedance as expressed in (3).

$$
Z_{\mathbf{\square}, \text { rough }}=-\mu_{0} \frac{E_{z}}{B_{y}}=-j \omega \frac{\int_{\sigma>0} B_{y} d x}{\int_{\sigma>0} J_{z} d x}
$$

\section{OPTIMISATIONS}

The mode-matching techniques are intrinsically computationally intensive operations and with the inclusion of a more rigorous treatment of the bounding walls the computational load is further increased. It is therefore necessary to consider where optimisation to the method can be made before modelling complete waveguide structures. One obvious gain is to derive the analytical expressions for the mode-matching equations, including the extensions to model the lossy elements. By using these analytic solutions significant reduction $(\sim 100$ times $)$ in simulation time can be achieved (Table 1). However, for some geometrical cases there exist no such solutions, i.e., offset cylindrical sections. Further optimisations can be made by partitioning the mode sets to avoid unnecessary computation where the results are known from the symmetry conditions of the structure Lastly, by reducing the familiar surface integrals at the junction between two guide sections to that of a contour integral [6], some performance gains can be achieved. These methods are prototyped in Python as such there are obvious gains from redevelopment in $\mathrm{C}++$.

As mentioned, solutions for cylindrical guide structures with non-concentric discontinuities are not generally solvable analytically. Some progress was made by considering small offsets applied to the junctions in [7], but typically we rely on numerical approaches to solve these cases. General mode-matching methods may be parallelised and implemented in OpenCL to take advantage of hardware acceleration in the form of an AMD W9100 FirePro GPU. Due to the nature of the problem, parallelisation through OpenCL provides a tremendous reduction in simulation time versus its linear counterpart; often approaching the analytics times where larger mode sets are considered With these optimisations in place the construction of solutions for the more complex conical and corrugated horn systems with finite wall conductivity may be achieved in finite time.

Table 1. Contrasts the different integration times for analytical, numerical and OpenCL implementations (NA - Not Applicable, TLTC — To Long To Compute).

\begin{tabular}{c|c|c|c}
\hline Integral Type & $\begin{array}{c}\text { Numerical } \\
\text { Time (s)/Cost (\%) }\end{array}$ & $\begin{array}{c}\text { Analytical } \\
\text { Time (s)/Cost (\%) }\end{array}$ & $\begin{array}{c}\text { OpenCL } \\
\text { Time (s)/Cost (\%) }\end{array}$ \\
\hline PEC (concentric) & & & \\
\hline 48 Modes & $1.21 \mathrm{~s} / 46.39 \%$ & $0.015 \mathrm{~s} / 1.03 \%$ & $0.052 \mathrm{~s} / 3.82 \%$ \\
\hline 200 Modes & $15.67 \mathrm{~s} / 83.21 \%$ & $0.072 \mathrm{~s} / 2.33 \%$ & $0.20 \mathrm{~s} / 4.81 \%$ \\
\hline PEC (Offset) & & & $0.051 \mathrm{~s} / 3.74 \%$ \\
\hline 48 Modes & $14.22 \mathrm{~s} / 91.28 \%$ & NA & $0.196 \mathrm{~s} / 4.78 \%$ \\
\hline 200 Modes & $482.66 \mathrm{~s} / 99.18 \%$ & NA & $0.635 \mathrm{~s} / 28.9 \%$ \\
\hline Lossy (concentric) & & & $1.54 \mathrm{~s} / 30.83 \%$ \\
\hline 48 Modes & $4.95 \mathrm{~s} / 76.63 \%$ & $0.063 \mathrm{~s} / 4.43 \%$ & \\
\hline 200 Modes & $54.52 \mathrm{~s} / 93.21 \%$ & $0.33 \mathrm{~s} / 9.02 \%$ & $0.165 \mathrm{~s} / 11.09 \%$ \\
\hline Lossy (Offset) & & & $0.541 \mathrm{~s} / 10.56 \%$ \\
\hline 48 Modes & $49.49 \mathrm{~s} / 97.32 \%$ & $\mathbf{N A}$ & \\
\hline 200 Modes & TLTC & NA &
\end{tabular}




\section{MODELLING COMPLETE GEOMETRIES}

As discussed in [2], by considering the surface impedance of the boundary walls we expect there to be losses on the modal fields which exist in the waveguide. By definition these losses are caused by induced surface currents produced via interaction between the modes magnetic field and the bounding wall. These losses are expected to manifest themselves more significantly in higher order modes, as field distributions for increasing azimuthal order modes are localised to a greater extent at the boundary walls. For multi-moded operation of guide structures the treatment of these losses will have to be considered in order to accurately describe the structure. As shown in Fig. 5, the fundamental mode for a cylindrical guide $\left(\mathbf{T E}_{\mathbf{1 1}}\right)$ has the majority of its field localised in the centre of the structure, whereas the higher order mode $\left(\mathbf{T E}_{\mathbf{3 1}}\right)$ is present more towards the bounding walls.
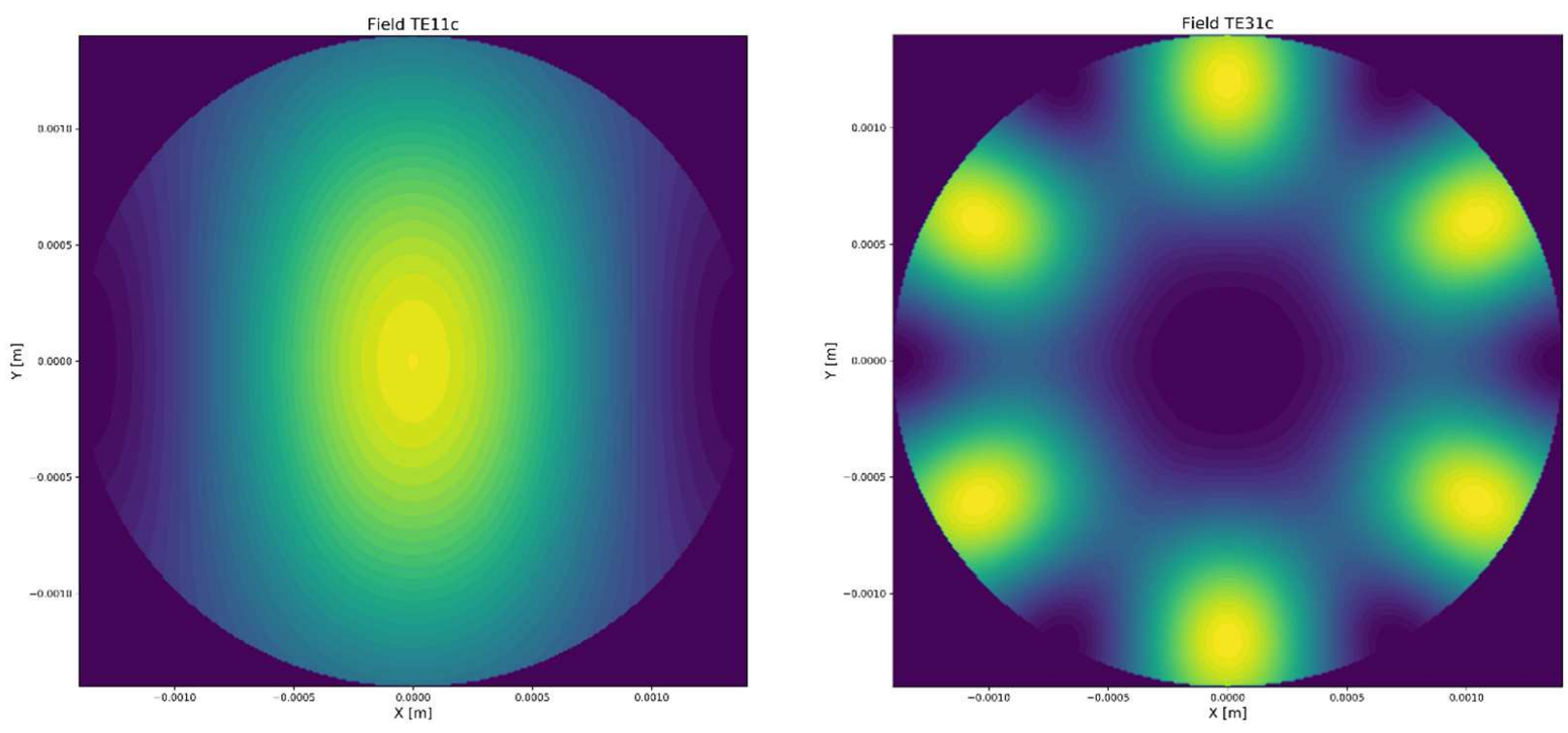

Figure 5. Contrast of the field distribution for the fundamental $\left(\mathrm{TE}_{11}\right)$ and higher order $\left(\mathrm{TE}_{31}\right)$ mode.

This effect can be demonstrated by considering a multi-moded cylindrical guide section with finite wall conductivity. Here we investigate the effect of increasing the effective impedance on the bounding walls (via varying conductivity or surface roughness) on the modes which are supported in the structure. As observed in Fig. 6, we see that there are losses associated with all modes present in the structure. However, the losses are more pronounced on the higher order modes $\left(\mathbf{T E}_{\mathbf{3 1}}\right)$ compared to the fundamental mode $\left(\mathbf{T} \mathbf{E}_{\mathbf{1 1}}\right)$ as there is a dramatic decrease in the ratio of $P_{\text {out }}: P_{\text {in }}$ as the impedance grows.

By considering these effects on multi-moded structures we aim to more accurately characterise their behaviour and aid in their design. Next we will see the effect of considering these losses on the far-field patterns of complete waveguide structures. The structures described in this section are both designed for use in CMB (cosmic microwave background) experiments. At their respective frequencies for multi-mode operation we define the structures with PEC and Lossy walls (conductivity $5.4 \times 10^{4} \mathrm{~S} / \mathrm{m}$ and roughness $1 \mu \mathrm{m}$ ). The extreme values for loss parameters are chosen to exaggerate the effect of losses in the guides. The influences of higher order modes are examined by considering the Co $\backslash$ Cross polarisation far-field components for the PEC and Lossy implementations of both structures. These figures are normalised to the co-polar component of the PEC field in all cases.

\subsection{Conical Horn (Spline Design)}

The conical test case corresponds to a Spline type design (Fig. 7), developed as one focal plane solution for the proposed COrE (Cosmic Origins Explorer) project. This is a single band device with an operating frequency range between $85-115 \mathrm{GHz}$ (Single Moded), with the spline shape optimised for Gaussicity and low return loss at $100 \mathrm{GHz}$. However, by increasing the frequency to $130 \mathrm{GHz}$ multi-moded operation can be achieved. A more complete description of this device is given in [1]. We model this guide at its purely single and multi-moded operational frequencies $(85 \mathrm{GHz}$ and $130 \mathrm{GHz})$ using the PEC and Lossy parameters defined earlier. 

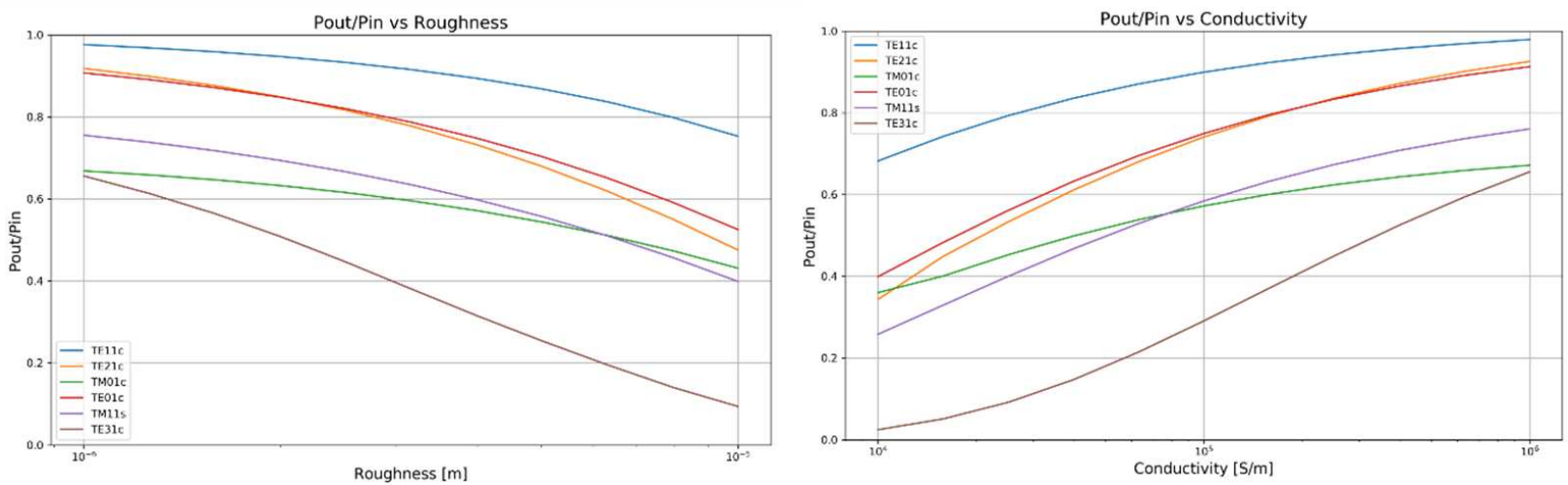

Figure 6. Varying of surface roughness or conductivity results in dramatic losses in higher order modes.

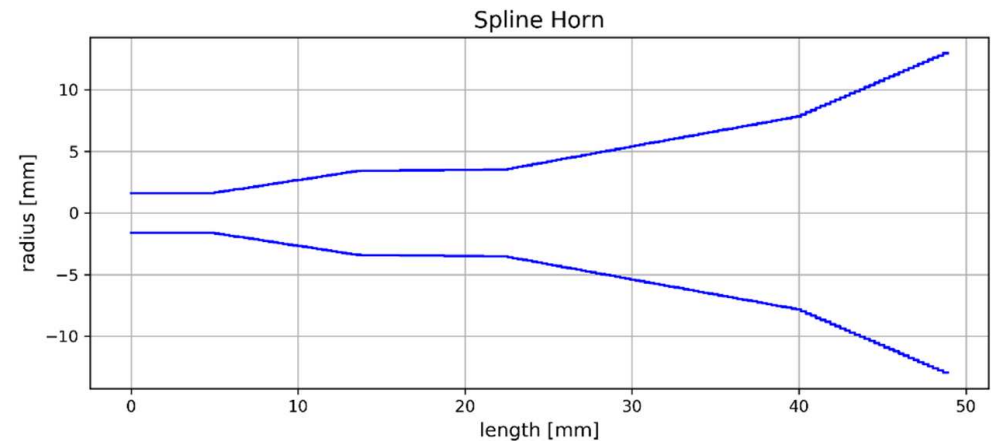

Figure 7. Visualisation of the spline horn structure.
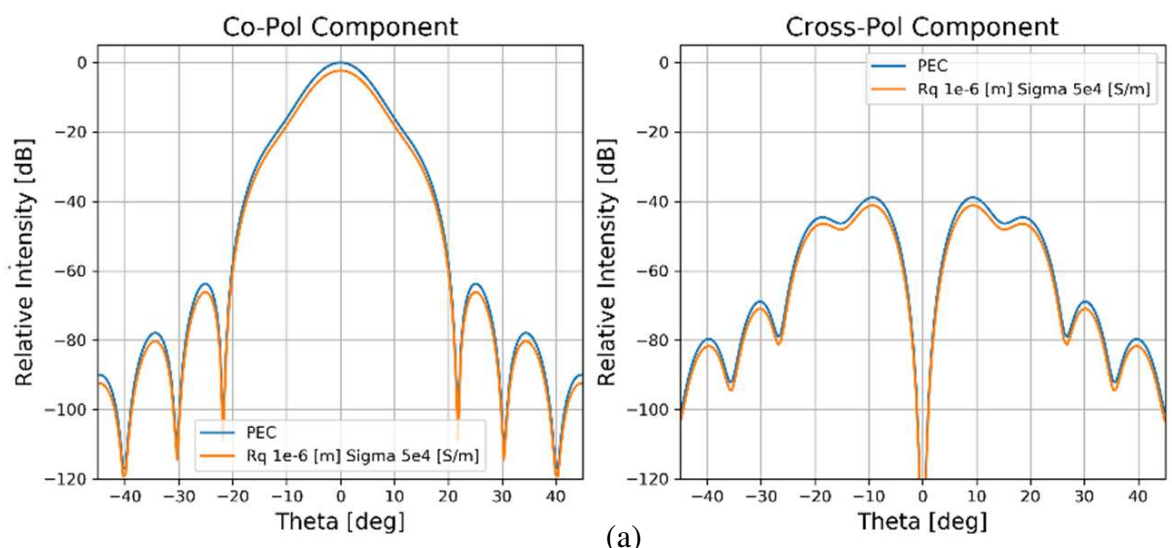

(a)
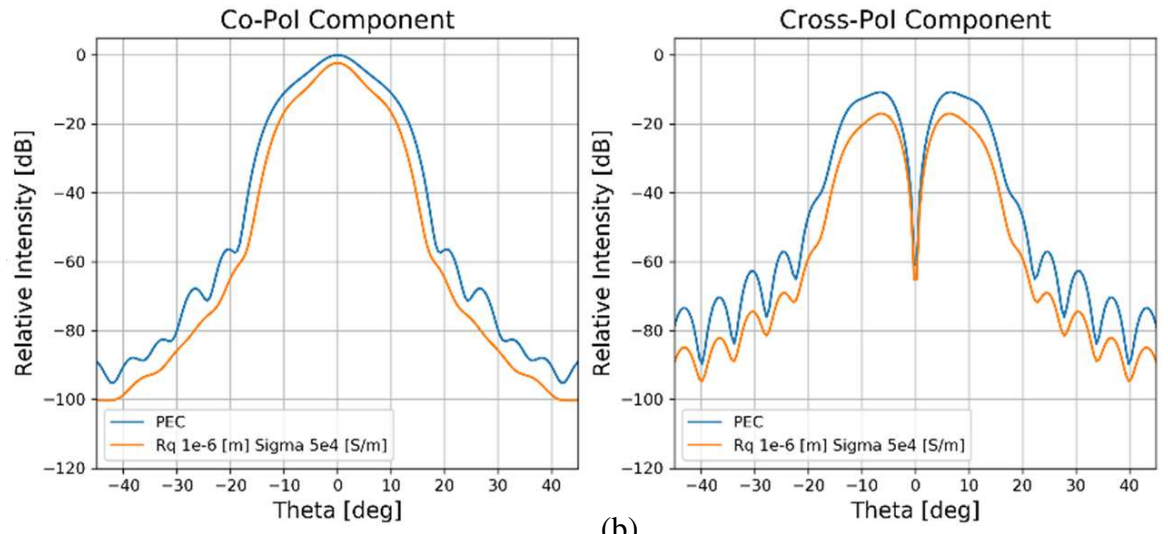

(b)

Figure 8. Shows the co-polar and cross-polar levels for the PEC and lossy results for the spline horn at (a) $85 \mathrm{GHz}$ (b) $130 \mathrm{GHz}$ with 10 azimuthal orders considered in each simulation. 
As observed in Fig. 8(a), the single moded operation of the conical guide is perturbed slightly by the inclusion of such a poor boundary wall. This is expected, since the fundamental (TE $\left.\mathbf{T}_{11}\right)$ modes' field resides mainly in the centre of the guide. Some losses are expected as it is not entirely localised and this confirms the results observed in Fig. 6. The multi-moded case presented, Fig. 8(b), sees 10 modes (including orthogonal modes) supported in this structure. In the PEC case this gives rise to a much wider co-polar component and an increase in the cross-polar levels due to the inclusion of the higher order modes [8]. However, in the lossy cases these effects are suppressed as the contribution from the higher order modes is negated by their interaction with the boundary wall. This results in a much narrower and reduced power in co-polar component along with diminished cross-polar levels.

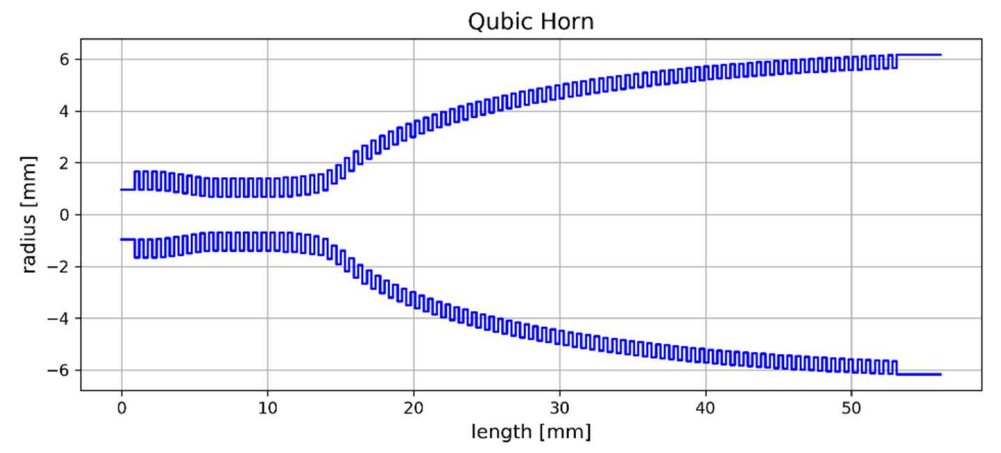

Figure 9. Visualisation of the QUBIC horn structure.
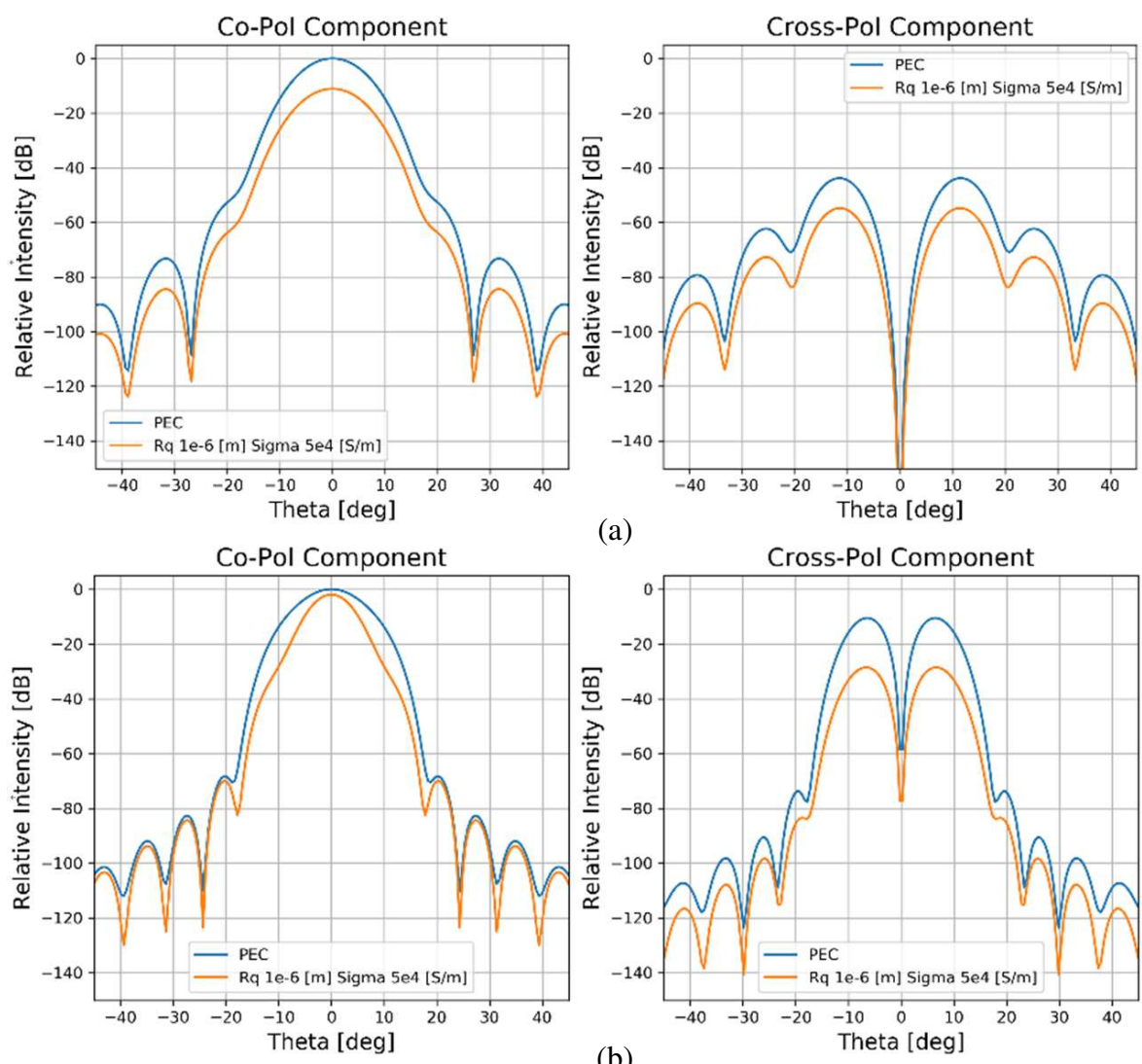

(a)

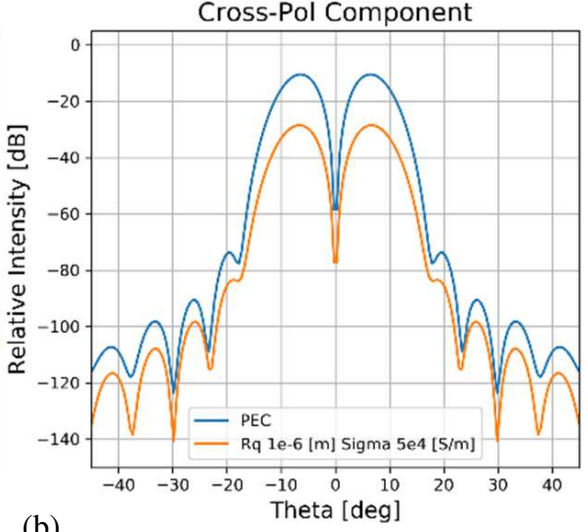

(b)

Figure 10. Shows the co-polar and cross-polar levels for the PEC and Lossy results for the QUBIC horn at (a) $145 \mathrm{GHz}$ and (b) $220 \mathrm{GHz}$ with 10 azimuthal orders considered in each simulation. 


\subsection{Corrugated Horn Structure (QUBIC Design)}

The corrugated test case corresponds to the QUBIC horn, which is a dual-band device with an operating range between 120-240 GHz (single moded 120-170 GHz, multi-moded 200-240 GHz). A detailed description of the QUBIC design, 4.2 is given in [9]. Again, we model this guide at its central single and multi-moded operational frequencies (145 GHz and $220 \mathrm{GHz})$ using the PEC and Lossy parameters defined earlier.

For single moded operation of corrugated horns the aperture field is required to be linear in structure and is supported only by the formation of a hybrid mode $\left(\mathbf{H E}_{\mathbf{1 1}}\right)$. This results in a symmetric axial beam with low cross-polar levels [10]. As observed in Fig. 10(a) we see that the resulting beam pattern has these required characteristics By considering the lossy walls there is a substantial reduction in the co-polar levels while its shape matches that of the PEC case. This is likely due to the suppression of the modes required in the formation of the fundamental hybrid mode of the structure. In general, for the multi-moded corrugated structures, the cross-polar levels increase with the inclusion of higher order modes as seen in Fig. 10(b). Similar behaviour to the conical case is observed, the lossy cases causes a narrowing of the co-polar beam and a reduction in the cross-polar levels since the higher order contributions are suppressed by the losses on the walls.

\section{CONCLUSION}

By considering the losses associated with conductivity and roughness parameters of the bounding walls for waveguide structures the potentially significant losses associated with the boundary walls could be demonstrated. For multi-moded operations it was observed that the inclusion of the lossy walls sufficiently suppressed the higher order contributions when compared to the PEC case. This type on analysis is of particular important to future millimetre/sub-millimetre measurement campaigns as the effects of guide imperfections will become more important with increasing frequency and mode order. The associated imperfections may arise from manufacturing techniques, such as spark erosion, which can damage the materials surface leading to an increase in impedance.

\section{ACKNOWLEDGMENT}

This research is funded under the Irish Research Council's Government of Ireland Postgraduate Scholarship.

\section{REFERENCES}

1. McCarthy, D., "Investigation of horn optimisation, simulation and measurement techniques, with application to integrating cavity based pixel design," Apr. 17, 2014.

2. Shafii, J. and R. J. Vernon, "Investigation of mode coupling due to ohmic wall losses in overmoded uniform and varying-radius circular waveguides by the method of cross sections," Vol. 50, No. 5, 1361-1369, 2002.

3. Wade, J. and R. H. Macphie, "Conservation of complex power technique for waveguide junctions with finite wall conductivity," Microw. Theory Tech., Vol. 38, No. 4, 1990.

4. Balanis, C. A., Advanced Engineering Electromagnetics, 2nd Edition, Wiley, 2012.

5. Gold, G. and K. Helmreich, "A physical surface roughness model and its applications," Vol. 65, No. 10, 3720-3732, 2017.

6. Figlia, G. and G. Gentili, "On the line-integral formulation of mode-matching technique," Microw. Theory Tech., Vol. 50, No. 2, 578-580, 2002.

7. Peacocke, P. T., "The modelling of scattering in corrugated waveguides," May 2012.

8. Olver, A. D., P. J. Clarricoats, A. A. Kishk, and L. Shafai, Microwave Horns and Feeds, 2011.

9. Scully, S., "Quasi-optical design and analysis of bolometric interferometers for cosmic microwave background experiments," Dep. Exp. Phys., No. 2583, 374, 2010.

10. Olver, A. D., L. Shafai, A. A. Kishk, P. J. Clarricoats, and A. Olver, "Corrugated horns," Microwave Horns and Feeds, 2011. 